\title{
Perturbative and non-perturbative aspects of the proper time renormalization group
}

\author{
D. Zappalà \\ INFN, Sezione di Catania \\ Dipartimento di Fisica, Università di Catania \\ Corso Italia 57, I-95129, Catania, Italy
}

\begin{abstract}
The renormalization group flow equation obtained by means of a proper time regulator is used to calculate the two loop $\beta$-function and anomalous dimension $\eta$ of the field for the $O(N)$ symmetric scalar theory. The standard perturbative analysis of the flow equation does not yield the correct results for both $\beta$ and $\eta$. We also show that it is still possible to extract the correct $\beta$ and $\eta$ from the flow equation in a particular limit of the infrared scale. A modification of the derivation of the Exact Renormalization Group flow, which involves a more general class of regulators, to recover the proper time renormalization group flow is analyzed.
\end{abstract}

Pacs 11.10.Gh, 11.10.Hi 


\section{Introduction}

The description of critical phenomena and, in general, of phenomena which involve the physics of many scales at the same time, is successfully achieved by means of the Renormalization Group techniques developed on the basis of the work of Wilson [1]. Recently these techniques have been refined by many authors and a particular version of the flow equation, generally indicated as Exact Renormalization Group (ERG) has been formulated [2, 3, 4, 5, 6, 7]. Basically this flow is called exact because it is formally derived from the generator of the Green functions of the theory, without explicitly performing the functional integration and without resorting to any specific approximation or truncation. Many details and applications of this flow equation can be found for instance in [8, 9].

Beside the ERG, other formulations of the renormalization group flow have been derived. In particular we shall focus on one of them which is known as Proper Time Renormalization Group (PTRG) and which has been formulated and studied in many applications [10, 11, 12, 13, 14, 15, 16, 17, 18, 19]. This flow equation is obtained by making use of the Proper Time representation 20] and by introducing a suitable multiplicative regulator as a cut-off on the Infrared (IR) modes. It must be remarked that, substantially, the PTRG is obtained in the quoted papers as a renormalization group improvement of the one loop effective action.

Due to the lack of a formal proof of exactness similar to the one concerning the ERG and due to its straightforward interpretation in terms of improvement on the one loop calculation, many doubts have been cast about the complete reliability of the results obtained from the PTRG equation. On the other hand there are some examples where this equation provided reliable results. In fact the derivative expansion truncated to the lowest order (local potential approximation) and to the first order (which consists of two coupled flow equations for the potential $U_{k}$ and for the wave function renormalization $Z_{k}$ ) has been employed to determine some critical exponents at the non-gaussian fixed point of the scalar theory in three dimensions [14, 15]. These coupled flow equations have been used for another application in quantum mechanics, namely the determination of the energy gap between the ground state and the first excited state of the double well potential [16], where the exact value can be numerically evaluated and therefore a quantitative check of the flow equations is possible. Obviously these results determined by means of the PTRG show a dependence 
on the form of the proper time regulator considered and it turned out that the optimal choice corresponds to the sharp limit of this regulator. In this limit the flow equations for $U_{k}$ and $Z_{k}$ have a simple form and the various quantities determined are certainly comparable to the ones extracted from the ERG.

Then it would be interesting to analyze the relation between this flow and the ERG. This issue has already been addressed in [17, 18, 19]. In this paper we focus both on some properties of the perturbative expansion of the PTRG and on the possibility of introducing a cut-off on the ultraviolet modes for the PTRG, with the same procedure employed for the ERG. Before illustrating the details of our analysis we examine some points concerning the Proper Time flow equation and the sharp limit on the regulator which has been mentioned above. The Proper Time flow equation used in [16] is

$$
k \partial_{k} \Gamma_{k}=-\frac{1}{2} \operatorname{Tr} \int_{0}^{\infty} \frac{\mathrm{d} s}{s}\left(k \partial_{k} f_{k}\right) \exp \left(-s \frac{\delta^{2} \Gamma_{k}}{\delta \varphi \delta \varphi}\right)
$$

where the derivative of the regulator $k \partial_{k} f_{k}$ has the form

$$
k \partial_{k} f_{k}=-2 s m Z_{k} k^{2} \exp \left(-s m Z_{k} k^{2}\right) \frac{\left(s m Z_{k} k^{2}\right)^{m}}{m !}
$$

and $Z_{k}$ is the wave function renormalization coefficient of the kinetic term $(\partial \varphi)^{2}$ of the running effective action $\Gamma_{k}$ and $m$ is a positive integer. The regulator considered here depends on the particular value assigned to the index $m$ and, as discussed before it has been checked that in the various applications the results are optimized in the sharp limit which corresponds to $m \rightarrow \infty$ [14] (incidentally we notice that a slightly different form of $k \partial_{k} f_{k}$ has been used in [14, 15] and by other authors, which however is less suitable to study the limit $m \rightarrow \infty)$. In [16] the flow equation for $U_{k}$ and $Z_{k}$ were obtained in this limit but here we are interested in the form of the full flow equation. To analyze the large $m$ limit we follow the derivation outlined in [18. We replace the factorial in Eq. (2) by making use of the Stirling formula $(m-1)$ ! = $e^{-m} m^{m} \sqrt{(2 \pi / m)}(1+O(1 / m))$ and neglecting the terms that vanish in the limit $m \rightarrow \infty$ we get

$$
k \partial_{k} f_{k}=-2 s Z_{k} k^{2} \exp \left[-m\left(s Z_{k} k^{2}-\ln \left(s Z_{k} k^{2}\right)-1\right)\right] \sqrt{\frac{m}{2 \pi}}
$$

The limit $m \rightarrow \infty$ then yields

$$
\begin{aligned}
& \lim _{m \rightarrow \infty} k \partial_{k} f_{k}^{m}=-\sqrt{2} s Z_{k} k^{2} \delta\left(\sqrt{s Z_{k} k^{2}-\ln \left(s Z_{k} k^{2}\right)-1}\right) \\
& =-2 s \delta\left(s-\frac{1}{Z_{k} k^{2}}\right)
\end{aligned}
$$


where, in the first step we have introduced the $\delta$-function according to

$$
\lim _{\epsilon \rightarrow 0} \frac{\exp \left(-x^{2} / \epsilon^{2}\right)}{\epsilon \sqrt{\pi}}=\delta(x)
$$

and with the position $m=1 / \epsilon^{2}$. The result in Eq. (4) directly leads to the full flow equation in the large $m$ limit which, according to Eq. (丑), reads

$$
k \frac{\partial \Gamma_{k}}{\partial k}=\operatorname{Tr}\left\{\exp \left(-\frac{1}{Z_{k} k^{2}} \frac{\delta^{2} \Gamma_{k}}{\delta \varphi \delta \varphi}\right)\right\}
$$

Eq. (6) is the starting point of our analysis and in the following we indicate this flow equation as the PTRG flow. Clearly the derivative expansion of Eq. (6) yields again those flow equation for $U_{k}$ and $Z_{k}$ which have been analyzed in [15, 16].

In Section 2 we consider a purely perturbative calculation and we evaluate by means of the flow equation the two loop anomalous dimension of the field and $\beta$-function for the $O(N)$ symmetric scalar theory in four dimensions, which, as it is well known, correspond respectively to the $O\left(\lambda^{2}\right)$ and $O\left(\lambda^{3}\right)$ terms in the expansion in powers of the quartic coupling $\lambda$. This calculation has already been performed by making use of the ERG [21], but it is interesting to reconsider it within the PTRG framework because of the recent analysis in [19 where it is argued that the proper time flow equation does not respect the diagrammatic structure beyond one loop and should therefore produce wrong predictions within a perturbative expansion. We develop a systematic procedure to calculate the one-particle irreducible (1PI) vertices in the perturbative expansion and show that the correct two loop anomalous dimension and $\beta$ function are not reproduced by the standard perturbative analysis of Eq. (6). However we also discuss a particular limit of the flow equation in which the correct two loop quantities are recovered. In Section 3 we follow the procedure outlined in [4] to derive the ERG flow and try to reformulate it in the case of the PTRG. Finally, Section 4 contains some comments about the results obtained and the conclusions.

\section{The two loop $\beta$-function}

In this Section we consider the perturbative analysis of Eq. (6) and, in particular, we discuss the computation of the anomalous dimension $\eta$ and the 
$\beta$-function of the $O(N)$ scalar theory respectively to order $O\left(\lambda^{2}\right)$ and $O\left(\lambda^{3}\right)$ of their expansion in powers of the quartic coupling constant $\lambda$, which correspond to the two loop calculation of these quantities. We shall check that the usual correct perturbative procedure fails at two loop but at the same time we show that the two loop $\beta$ and $\eta$ are recovered from Eq. (6) in a specific limit.

To this aim we need the coupled flow equations for various 1PI vertices which, in turn, must be obtained from the full equation for $\Gamma_{k}$ by functional derivation. Once the set of (infinite) coupled flow equations for the 1PI vertices is determined, it can be solved within a particular truncation which is dictated by the specific order in the perturbative series one is interested in.

The first step concerns the functional derivation of the full flow equation which involves an exponential term. The latter must be properly expanded in order to avoid commutation problems when taking the derivatives (in the following we can safely neglect the factor $1 / Z_{k}=1+O(\lambda)$ which appears in the exponential in Eq. (6) because it does not give any contribution to the perturbative order we are interested in). Therefore it is convenient to disentangle in $\left(\delta^{2} \Gamma_{k} / \delta \phi \delta \phi\right)$ the field independent and the field dependent parts which we call respectively $A$ and $B$ :

$$
\frac{\delta^{2} \Gamma_{k}}{\delta \phi \delta \phi}=A+B
$$

and then the required expansion, which has been derived in [20], is

$$
\begin{aligned}
& \operatorname{Tr}\left\{\exp \left(-\frac{A+B}{k^{2}}\right)\right\}=\operatorname{Tr}\left\{\exp \left(-\frac{A}{k^{2}}\right)\right\}-\frac{1}{k^{2}} \operatorname{Tr}\left\{B \cdot \exp \left(-\frac{A}{k^{2}}\right)\right\} \\
& +\frac{1}{2 k^{4}} \operatorname{Tr}\left\{\int_{0}^{1} \mathrm{~d} u\left[B \cdot \exp \left(-\frac{A}{k^{2}}(1-u)\right) \cdot B \cdot \exp \left(-\frac{A}{k^{2}} u\right)\right]\right\} \\
& +\ldots+\frac{(-1)^{n+1}}{(n+1) k^{2 n+2}} \operatorname{Tr}\left\{\int _ { 0 } ^ { 1 } \mathrm { d } u _ { 1 } \ldots \int _ { 0 } ^ { 1 } \mathrm { d } u _ { n } \left[u_{1}^{n-1} u_{2}^{n-2} \ldots u_{n-1}^{1} u_{n}^{0}\right.\right. \\
& B \cdot \exp \left(-\frac{A}{k^{2}}\left(1-u_{1}\right)\right) \cdot B \cdot \exp \left(-\frac{A}{k^{2}} u_{1}\left(1-u_{2}\right)\right) \cdot B \\
& \cdot \exp \left(-\frac{A}{k^{2}} u_{1} u_{2}\left(1-u_{3}\right)\right) \cdot B \cdot \ldots \cdot B \cdot \exp \left(-\frac{A}{k^{2}} u_{1} u_{2} \ldots u_{n-1}\left(1-u_{n}\right)\right) \\
& \left.\left.\cdot B \cdot \exp \left(-\frac{A}{k^{2}} u_{1} u_{2} \ldots u_{n-1} u_{n}\right)\right]\right\}+\ldots
\end{aligned}
$$

In Eq. (8) only the field independent part $A$ appears in the exponentials and it is now simple to take the functional derivatives of this expression where the 
field dependence is all contained in $B$. The $n$-point $1 \mathrm{PI}$ function is obtained by evaluating the functional derivative of order $n$ of Eq. (8) for vanishing fields. Moreover, due to the splitting in Eq. (7), in a perturbative expansion one expects that $A$ contains the lowest order term which is not proportional to the coupling constant whereas the field dependent part $B$ is a $O(\lambda)$ term and therefore Eq. (8) naturally provides an expansion in powers of the coupling constant.

For our purpose we shall consider the case of the quartic massless $O(N)$ scalar theory in four dimensions with Euclidean action

$$
S=\int \mathrm{d}^{4} x\left(\frac{1}{2} \partial \phi_{i} \partial \phi_{i}+\frac{\lambda}{4 !}\left(\phi_{i} \phi_{i}\right)^{2}\right)
$$

Due to the symmetry, the $n$-point 1 PI functions with odd $n$ are zero to each order of the perturbative expansion. Therefore from Eq. (8) one gets the following flow equations in the momentum space for the two, four and sixpoint functions

$$
k \frac{\partial \Gamma_{i, j}^{(2)}(q,-q)}{\partial k}=-\frac{1}{k^{2}} \operatorname{Tr}_{\mathrm{t}}\left\{\Gamma_{i, j, l_{1}, l_{2}}^{(4)}(q,-q, t,-t) D_{l_{2}, l_{1}}^{1}(t)\right\}
$$

$$
\begin{aligned}
& k \frac{\partial \Gamma_{i, j, r, s}^{(4)}(q,-q, p,-p)}{\partial k}=\frac{1}{k^{4}} \operatorname{Tr}_{\mathrm{t}}\left\{\int_{0}^{1} \mathrm{~d} u_{1}\right. \\
& \times\left[\Gamma_{i, r, l_{1}, l_{2}}^{(4)}(q, p, t,-q-p-t) D_{l_{2}, l_{3}}^{1-u_{1}}(q+p+t) \Gamma_{j, s, l_{3}, l_{4}}^{(4)}(-q,-p, q+p+t,-t) D_{l_{4}, l_{1}}^{u_{1}}(t)\right. \\
& +\Gamma_{j, r, l_{1}, l_{2}}^{(4)}(-q, p, t, q-p-t) D_{l_{2}, l_{3}}^{1-u_{1}}(-q+p+t) \Gamma_{i, s, l_{3}, l_{4}}^{(4)}(q,-p,-q+p+t,-t) D_{l_{4}, l_{1}}^{u_{1}}(t) \\
& \left.\left.+\Gamma_{i, j, l_{1}, l_{2}}^{(4)}(q,-q, t,-t) D_{l_{2}, l_{3}}^{1-u_{1}}(t) \Gamma_{r, s, l_{3}, l_{4}}^{(4)}(p,-p, t,-t) D_{l_{4}, l_{1}}^{u_{1}}(t)\right]\right\} \\
& -\frac{1}{k^{2}} \operatorname{Tr}_{\mathrm{t}}\left\{\Gamma_{i, j, r, s, l_{1}, l_{2}}^{(6)}(q,-q, p,-p, t,-t) D_{l_{2}, l_{1}}^{1}(t)\right\}
\end{aligned}
$$




$$
\begin{aligned}
& k \frac{\partial \Gamma_{i, j, r, s, w, z}^{(6)}(0,0,0,0, q,-q)}{\partial k}=-\frac{1}{3 k^{6}} \operatorname{Tr}_{\mathrm{t}}\left\{\int_{0}^{1} \mathrm{~d} u_{1} u_{1} \int_{0}^{1} \mathrm{~d} u_{2}\right. \\
& \times\left[3 \mathcal { P } _ { ( i , j , r , s ) } \left(\Gamma_{w, z, l_{1}, l_{2}}^{(4)}(q,-q, t,-t) D_{l_{2}, l_{3}}^{1-u_{1}}(t) \Gamma_{i, j, l_{3}, l_{4}}^{(4)}(0,0, t,-t)\right.\right. \\
& \left.\times D_{l_{4}, l_{5}}^{u_{1}\left(1-u_{2}\right)}(t) \Gamma_{r, s, l_{5}, l_{6}}^{(4)}(0,0, t,-t) D_{l_{6}, l_{1}}^{u_{1} u_{2}}(t)\right) \\
& +12 \mathcal{P}_{(i, j, r, s)}\left(\Gamma_{w, i, l_{1}, l_{2}}^{(4)}(q, 0, t,-q-t) D_{l_{2}, l_{3}}^{1-u_{1}}(q+t) \Gamma_{z, j, l_{3}, l_{4}}^{(4)}(-q, 0, q+t,-t)\right. \\
& \times D_{l_{4}, l_{5}}^{u_{1}\left(1-u_{2}\right)}(t) \Gamma_{r, s, l_{5}, l_{6}}^{(4)}(0,0, t,-t) D_{l_{6}, l_{1}}^{u_{1} u_{2}}(t) \\
& +\Gamma_{w, i, l_{1}, l_{2}}^{(4)}(q, 0, t,-q-t) D_{l_{2}, l_{3}}^{1-u_{1}}(q+t) \Gamma_{r, s, l_{3}, l_{4}}^{(4)}(0,0, q+t,-q-t) \\
& \times D_{l_{4}, l_{5}}^{u_{1}\left(1-u_{2}\right)}(q+t) \Gamma_{z, j, l_{5}, l_{6}}^{(4)}(-q, 0, q+t,-t) D_{l_{6}, l_{1}}^{u_{1} u_{2}}(t) \\
& +\Gamma_{r, s, l_{1}, l_{2}}^{(4)}(0,0, t,-t) D_{l_{2}, l_{3}}^{1-u_{1}}(t) \Gamma_{w, i, l_{3}, l_{4}}^{(4)}(q, 0, t,-q-t) \\
& \left.\times D_{l_{4}, l_{5}}^{u_{1}\left(1-u_{2}\right)}(q+t) \Gamma_{z, j, l_{5}, l_{6}}^{(4)}(-q, 0, q+t,-t) D_{l_{6}, l_{1}}^{u_{1} u_{2}}(t)\right) \\
& \left.\left.+12 \mathcal{P}_{(i, j, r, s)}((w, q) \leftrightarrow(z,-q))\right]\right\}+H . O . T .
\end{aligned}
$$

where the sum over repeated internal space indices is understood and, for simplicity, the dependence of the 1PI functions on the scale $k$ is not indicated explicitly and the following notations have been used

$$
\begin{gathered}
D_{i, j}^{y}(p)=\exp \left(-\frac{\Gamma_{i, j}^{(2)}(p,-p)}{k^{2}} y\right) \\
\operatorname{Tr}_{\mathrm{t}}=\int \frac{\mathrm{d}^{4} t}{(2 \pi)^{4}}
\end{gathered}
$$

In Eq. (12) the symbol $\mathcal{P}_{(i, j, r, s)}$ indicates the sum over the permutations of the indices $i, j, r, s$ but excludes the permutations of the indices belonging to the same function $\Gamma^{(4)}$ (which would correspond to a double counting). Namely the first $\mathcal{P}_{(i, j, r, s)}$ in Eq. (12) corresponds to six possible permutations, the second one corresponds to twelve possible permutations, the third one is just like the second one but with both index $w$ and momentum $q$ simultaneously exchanged with $z$ and $-q$. Furthermore in Eq. (12) there is also a contribution 
of higher order terms, proportional to $\Gamma^{(6)}$ and $\Gamma^{(8)}$, indicated as H.O.T. which is negligible for the determination of the two loop $\beta$-function. Finally we note that in Eqs. (11, 12) only particular configurations of the momenta of $\Gamma^{(4)}$ and $\Gamma^{(6)}$ are considered. In fact these are the configurations which, as we shall see below, are relevant for the two loop $\beta$-function and anomalous dimension.

The perturbative computation is performed by expressing each $\Gamma^{(n)}$ as a series in powers of the coupling $\lambda$ and reading from Eq. (9) the lowest nonvanishing terms (which are indicated with a hat)

$$
\begin{gathered}
\widehat{\Gamma}_{i, j}^{(2)}(q,-q)=q^{2} \delta_{i j} \\
\widehat{\Gamma}_{i, j, r, s}^{(4)}\left(q_{1}, q_{2}, q_{3},-q_{1}-q_{2}-q_{3}\right)=\frac{\lambda}{3} \Delta(i, j, r, s)
\end{gathered}
$$

where $\delta_{i j}$ is the Kronecker delta and

$$
\Delta(i, j, r, s)=\left(\delta_{i j} \delta_{r s}+\delta_{i r} \delta_{j s}+\delta_{i s} \delta_{j r}\right)
$$

When Eqs. (15, 16) are inserted in the right hand side of the flow equations, one can recover the first order corrections to the $n$-point functions, which in turn can be put again in the flow equations to get the second order and so on. By collecting in each $n$-point function all contributions with a fixed power of the coupling constant one obtains the various orders of the perturbative expansion.

We shall first consider the anomalous dimension $\eta$, defined as

$$
\eta \delta_{i j}=-k \frac{\partial}{\partial k}\left(\left[\frac{\partial \Gamma_{i, j}^{(2)}(q,-q)}{\partial q^{2}}\right]_{q=0}\right)
$$

Clearly the lowest order in Eq. (15) does not give any contribution to $\eta$ and it is necessary to consider the first correction to $\Gamma^{(2)}$ in Eq. (10) which is obtained by replacing $\Gamma^{(4)}$ in the right hand side of Eq. (10) with its lowest order displayed in Eq. (16). However this correction to $\Gamma^{(2)}$ has no dependence on the external momentum of the two-point function and then it does not contribute to $\eta$. The first non-vanishing contribution is obtained by considering the first correction to $\Gamma^{(4)}$ in Eq. (10). This correction is to be computed from Eq. (11) and for this purpose the required configuration of the external momenta of $\Gamma^{(4)}$ is just the one considered in Eq. (11]). The lowest contribution coming from Eq. (11) is of order $O\left(\lambda^{2}\right)$. (Note that in Eq. (10) there is another contribution 
of order $O\left(\lambda^{2}\right)$ coming from the $O(\lambda)$ correction to $\Gamma^{(2)}$ in the exponential but it is independent of the external momentum and therefore irrelevant for $\eta$ ).

Our next step for the determination of $\eta$ is the computation of the $O\left(\lambda^{2}\right)$ terms in Eq. (11) which yield an external momentum dependence when inserted in Eqs. (10) and (18). This means that, of the three terms in Eq. (11) quadratic in $\Gamma^{(4)}$, the last one must be neglected. Furthermore, the term proportional to $\Gamma^{(6)}$ must be discarded because it is of order $O\left(\lambda^{3}\right)$ as it is evident from Eq. (12). Then we replace the lowest order terms (15) and (16) in the remaining part of Eq. (11) and then insert this in Eq. (10). From this procedure we do not get the full $\Gamma^{(2)}$ to the order $O\left(\lambda^{2}\right)$ but only the relevant part for determining the anomalous dimension, and we indicate it as $\Gamma^{(2) \eta}$ :

$$
\begin{aligned}
& k \frac{\partial \Gamma_{i, j}^{(2) \eta}(q,-q)}{\partial k}=-\delta_{i j} \frac{(N+2) \lambda^{2}}{3 k^{2}} \\
& \times \operatorname{Tr}_{\mathrm{p}}\left\{\operatorname { e x p } ( - \frac { p ^ { 2 } } { k ^ { 2 } } ) \int _ { \Lambda } ^ { k } \frac { \mathrm { d } x } { x ^ { 5 } } \operatorname { T r } _ { \mathrm { t } } \left\{\int_{0}^{1} \mathrm{~d} u_{1} \exp \left(-\frac{t^{2}}{x^{2}}\left(1-u_{1}\right)\right)\right.\right. \\
& \left.\left.\times\left[\exp \left(-\frac{(t+p+q)^{2}}{x^{2}} u_{1}\right)+\exp \left(-\frac{(t+p-q)^{2}}{x^{2}} u_{1}\right)\right]\right\}\right\}
\end{aligned}
$$

In Eq. (19) the factor $(N+2) / 3$ comes from the various sums over the internal indices and the integral on the variable $x$ corresponds to the integration of Eq. (11) to get $\Gamma^{(4)}$ at the scale $k$ (note that we are omitting the boundary value of $\Gamma^{(4)}$ at the Ultraviolet (UV) extremum $\Lambda$ because this bare value does not depend on the momentum $q$ and $\Lambda$ will be sent to infinity in this calculation because this does not introduce any UV divergences).

At this point the consistent determination of $\eta$ through Eq. (18) would require a direct resolution of the integrals in Eq. (19). This can be easily performed but it does not lead to the correct two loop coefficient of the squared coupling $\left(\lambda /\left(16 \pi^{2}\right)\right)^{2}$. For instance in the $N=1$ case instead of the correct $1 / 6$ coefficient, the value 0.1278 is obtained. However it is still possible to recover the two loop anomalous dimension from Eq. (19) in the following way. Let us consider the right hand side of (19). The integrand of the trace $\operatorname{Tr}_{p}$ has two factors: the vertex $\Gamma^{(4)}$ obtained from the corresponding flow equation and the exponential factor $\exp \left(-p^{2} / k^{2}\right)$. Before performing the integrals we consider the limit $k \rightarrow 0$ of these two separate terms in order to get the full contribution, up to $k=0$, of the four-point function to $\eta$, and we note that the exponential term in this limit is singular and becomes a $\delta$-function according to Eq. (囬) with the position $k=\epsilon$. When we replace the exponential with four 
$\delta$-functions, one for each component of the momentum, according to Eq. (5) we get

$$
\begin{aligned}
& k \frac{\partial \Gamma_{i, j}^{(2) \eta}(q,-q)}{\partial k}=-\delta_{i j} \frac{(N+2) \pi^{2} \lambda^{2}}{3} \\
& \times \operatorname{Tr}_{\mathrm{p}}\left\{\delta ^ { 4 } ( p ) k ^ { 2 } \int _ { \Lambda } ^ { k } \frac { \mathrm { d } x } { x ^ { 5 } } \operatorname { T r } _ { \mathrm { t } } \left\{\int_{0}^{1} \mathrm{~d} u_{1} \exp \left(-\frac{t^{2}}{x^{2}}\left(1-u_{1}\right)\right)\right.\right. \\
& \left.\left.\times\left[\exp \left(-\frac{(t+q)^{2}}{x^{2}} u_{1}\right)+\exp \left(-\frac{(t-q)^{2}}{x^{2}} u_{1}\right)\right]\right\}\right\}
\end{aligned}
$$

In Eq. (20) the limit $k \rightarrow 0$ of the exponential has already been performed but we still have to consider the same limit in the remaining $k$ dependent part of the equation and it is important to remark that the factor $k^{2}$ which appears explicitly in the trace in Eq. (20) cancels against a factor $\left(1 / k^{2}\right)$ coming from the $x$ integration, so that the final result is a finite number. The integrals over the momenta $p$ and $t$ are gaussian and they are easily performed and after that, according to Eq. (18), one can derive with respect to $q^{2}$ and then evaluate at $q=0$ and finally perform the remaining integrals with $\Lambda \rightarrow+\infty$. This procedure yields

$$
\eta=\left(\frac{\lambda}{16 \pi^{2}}\right)^{2} \frac{(N+2)}{18}
$$

Incidentally we note that the determination of the two loop anomalous dimension for the $N=1$ theory from the PTRG flow equation, by making use of the derivative expansion according to a procedure previously introduced in [22], has been already discussed in [15].

Let us now turn to the $\beta$-function. Again the correct perturbative procedure only provides the one loop $\beta$ and fails at two loop. Nevertheless the particular limit considered for the anomalous dimension works even in this case, as discussed below. The one loop $\left(O\left(\lambda^{2}\right)\right) \beta$-function is easily obtained from Eq. (11) evaluated at zero external momenta $q=p=0$ and replacing the four-point and two-point functions in the right hand side with their lowest order expressions in Eqs. (15) and (16). This yields

$$
k \frac{\partial \Gamma_{i, j, r, s}^{(4)}(0,0,0,0)}{\partial k}=\frac{(N+8) \lambda^{2}}{9 k^{4}} \Delta(i, j, r, s) \operatorname{Tr}_{\mathrm{t}}\left\{\exp \left(-\frac{t^{2}}{k^{2}}\right)\right\}
$$

As it has been done for the anomalous dimension, we evaluate the trace in Eq. (22) in the limit $k \rightarrow 0$ and make use of Eq. (5). The one loop $\beta$-function 
is then obtained from Eq. (22) by discarding the factor $\Delta(i, j, r, s) / 3$ which provides the normalization of the lowest order in Eq. (16):

$$
\beta^{1-l o o p}=\frac{\lambda^{2}}{16 \pi^{2}} \frac{(N+8)}{3}
$$

Incidentally we note that, to this level, the trace in Eq. (22) would have given the same output even if it were evaluated without taking the limit $k \rightarrow 0$. However when considering higher order calculations this property is no longer true.

The $O\left(\lambda^{3}\right)$ or two loop $\beta$-function is the sum of many contributions. In fact the $\beta$-function describes the evolution of the renormalized four point function $\Gamma^{(4) R}$ with vanishing external momenta (note that as in Eq. (23) the normalization factor $\Delta(i, j, r, s) / 3$ of the coupling in Eq. (16) is not included in the definition of the $\beta$-function )

$$
\beta \frac{\Delta(i, j, r, s)}{3}=k \frac{\partial \Gamma_{i, j, r, s}^{(4) R}(0,0,0,0)}{\partial k}=k \frac{\partial\left(Z_{0}^{-2} \Gamma_{i, j, r, s}^{(4)}(0,0,0,0)\right)}{\partial k}
$$

where $Z_{0}$ is the field independent part of the wave function renormalization $Z_{k}$ and it is obtained from the two-point function as

$$
Z_{0}=\left[\frac{\partial \Gamma_{i, i}^{(2)}(q,-q)}{\partial q^{2}}\right]_{q=0}
$$

The index $i$ in the right hand side is not summed and can be fixed arbitrarily due to the $O(N)$ symmetry. Then it is clear from Eq. (24) that there are two separate terms $\beta_{g}$ and $\beta_{\eta}$

$$
\begin{aligned}
& \beta \frac{\Delta(i, j, r, s)}{3}=\left(Z_{0}^{-2} \beta_{g}+\beta_{\eta}\right) \frac{\Delta(i, j, r, s)}{3} \\
& =Z_{0}^{-2} k \frac{\partial \Gamma_{i, j, r, s}^{(4)}(0,0,0,0)}{\partial k}-2 \Gamma_{i, j, r, s}^{(4)}(0,0,0,0) k \frac{\partial Z_{0}}{\partial k}
\end{aligned}
$$

and, since $Z_{0}=1+O\left(\lambda^{2}\right)$, as it follows from Eqs. (15), (18), (25), we see that, to order $O\left(\lambda^{2}\right)$ the $\beta$-function is simply given by $\beta_{g}$ which is what has been computed in Eq. (22). To order $O\left(\lambda^{3}\right)$ we have from Eqs. (25), (18) and (21)

$$
\beta_{\eta}=\frac{\lambda^{3}}{\left(16 \pi^{2}\right)^{2}} \frac{(N+2)}{9}
$$


We must compute now $\beta_{g}$ to order $O\left(\lambda^{3}\right)$ and we notice that its coefficient $Z_{0}^{-2}$ in Eq. (26) can be neglected because it gives contributions to $\beta$ which are at least of order $O\left(\lambda^{4}\right)$. Then, looking at the right hand side of Eq. (11) we can isolate three different contributions to $\beta_{g}$. The first one, which will be indicated as $\beta_{1}$, comes from the six-point function which is $O\left(\lambda^{3}\right)$, as Eq. (12) shows; the second one $\left(\beta_{2}\right)$ is originated by the terms quadratic in $\Gamma^{(4)}$ when one of these four-point functions is replaced with its first order expression $\left(O\left(\lambda^{2}\right)\right)$ and the other is kept to the lowest order; finally the last one $\left(\beta_{3}\right)$ comes from the same terms with both lowest order $\Gamma^{(4)}$ and considering the $O(\lambda)$ correction in the two-point functions appearing in the exponential factors.

The term $\beta_{1}$ requires the computation of $\Gamma^{(6)}$ for the particular external momenta displayed in Eq. (12). Then we insert Eqs. (15) and (16) into the right hand side of Eq. (12) and we also sum over the last two indices ( $w$ and $z$ ) because this is required when $\Gamma^{(6)}$ is inserted into Eq. (11)

$$
\begin{aligned}
& k \frac{\partial \Gamma_{i, j, r, s, w, z}^{(6)}(0,0,0,0, q,-q)}{\partial k} \delta_{w z} \\
& =-\frac{\lambda^{3}}{27 k^{6}} \Delta(i, j, r, s) \operatorname{Tr}_{\mathrm{t}} \int_{0}^{1} \mathrm{~d} u u\left\{2\left(N^{2}+10 N+16\right) \exp \left(-\frac{t^{2}}{k^{2}}\right)\right. \\
& \left.+8(5 N+22) \exp \left(-\frac{(t+q)^{2}}{k^{2}}(1-u)\right) \exp \left(-\frac{t^{2}}{k^{2}} u\right)\right\}
\end{aligned}
$$

The output of Eq. (28) must be used in Eq. (11) where we have the trace of $\Gamma^{(6)}$ times an exponential term. Again, before performing the trace we take the limit $k \rightarrow 0$ of these two terms and the exponential becomes a $\delta$-function on the four components of the momentum. Then it is straightforward to evaluate all the integrals and take the limit $\Lambda \rightarrow+\infty$ which yields

$$
\begin{aligned}
& \beta_{1}=-\frac{\lambda^{3} \pi^{2}}{9} \operatorname{Tr}_{\mathrm{p}}\left\{\delta ^ { 4 } ( p ) k ^ { 2 } \int _ { k } ^ { + \infty } \frac { \mathrm { d } x } { x ^ { 7 } } \operatorname { T r } _ { \mathrm { t } } \int _ { 0 } ^ { 1 } \mathrm { d } u u \left[2\left(N^{2}+10 N+16\right) \exp \left(-\frac{t^{2}}{x^{2}}\right)\right.\right. \\
& \left.\left.+8(5 N+22) \exp \left(-\frac{(t+p)^{2}}{x^{2}}(1-u)\right) \exp \left(-\frac{t^{2}}{x^{2}} u\right)\right]\right\}= \\
& =-\frac{\lambda^{3}}{\left(16 \pi^{2}\right)^{2}} \frac{\left(N^{2}+30 N+104\right)}{18}
\end{aligned}
$$

The procedure to calculate $\beta_{2}$ is analogous. The first step is to evaluate $\Gamma^{(4)}$ to order $O\left(\lambda^{2}\right)$ and the second step is to insert the result in Eq. (11). However in the second step we are interested in the limit $k \rightarrow 0$ which corresponds, as it 
has been done in the previous cases, to evaluate the $O\left(\lambda^{2}\right)$ four-point function with all vanishing external momenta, due to the presence of the $\delta$-function. Therefore we can directly evaluate the $\Gamma^{(4)}$ to order $O\left(\lambda^{2}\right)$ with all external momenta put to zero by integrating Eq. (11) from $\Lambda$ to $k$.

However we recall that the perturbative four-point function requires the introduction of a counterterm to cancel the divergences occurring in the computation. In fact with the renormalization condition that $\Gamma^{(4)}$ at zero external momenta is equal (apart from the normalization constant) to the coupling $\lambda$ : $\Gamma_{i, j, r, s}^{(4)}(0,0,0,0)=\lambda \Delta(i, j, r, s) / 3$, and by comparison with the lowest order prescription in Eq. (16), we see that this counterterm must cancel the $O\left(\lambda^{2}\right)$ contribution to the four-point function with vanishing external momenta, when the limits $\Lambda \rightarrow+\infty$ and $k \rightarrow 0$ are taken. Therefore in this case there is no contribution to the $\beta$-function, i.e.

$$
\beta_{2}=0
$$

Finally we consider $\beta_{3}$ which comes from the two-point function. We compute the $O(\lambda)$ correction to $\Gamma^{(2)}$ from Eq. (10). We get

$$
k \frac{\partial \Gamma_{i, j}^{(2)}(q,-q)}{\partial k}=-\frac{\lambda k^{2}}{16 \pi^{2}} \frac{(N+2)}{3} \delta_{i j}
$$

As for $\Gamma^{(4)}$ we need a counterterm which is fixed by the renormalization condition that the theory is massless. Therefore this counterterm must cancel exactly the term quadratic in $\Lambda$ coming from the integration of Eq. (31) so that we are left with a mass term proportional to $k^{2}$ which as required, is vanishing in the limit $k \rightarrow 0$. Therefore, once the quadratically divergent term has been cancelled, $\Gamma^{(2)}$ has the form $\Gamma_{i, j}^{(2)}(q,-q)=\left(q^{2}-\lambda(N+2) k^{2} /\left(6 \cdot 16 \pi^{2}\right)\right) \delta_{i j}$ and the exponential factor in Eq. (11) containing $\Gamma^{(2)}$ can be expanded in powers of the coupling and the $O(\lambda)$ term can be used in Eq. (11) to get $\beta_{3}$. This computation is straightforward

$$
\begin{aligned}
& \beta_{3}=\frac{(N+8) \lambda^{2}}{3 k^{4}} \operatorname{Tr}_{\mathrm{p}}\left\{\frac{\lambda(N+2)}{6\left(16 \pi^{2}\right)} \exp \left(-\frac{p^{2}}{k^{2}}\right)\right\} \\
& =\frac{\lambda^{3}}{\left(16 \pi^{2}\right)^{2}} \frac{(N+8)(N+2)}{18}
\end{aligned}
$$

where, again, we have taken the limit $k \rightarrow 0$ and reduced the exponential to a $\delta$-function and eventually performed the trace. 
Finally we collect all contributions to $\beta_{g}=\beta_{1}+\beta_{2}+\beta_{3}$ in Eqs. (29, 30, 32) and according to Eqs. (26) and (27) we get

$$
\beta^{2-\text { loop }}=\beta_{\eta}+\beta_{1}+\beta_{2}+\beta_{3}=-\frac{\lambda^{3}}{\left(16 \pi^{2}\right)^{2}} \frac{(3 N+14)}{3}
$$

Eqs. (21), (23) and (33) reproduce the correct perturbative anomalous dimension and $\beta$-function which had been already obtained by means of the ERG (e.g. see 21]).

Let us briefly comment on these results. The fact that the two loop $\beta$ function and anomalous dimension are not obtained from the standard perturbative analysis of Eq. (6) is in agreement with [19] where it is shown that the PTRG does not reproduce the two loop effective action. On the other hand we have seen that there exists a particular limit in which the flow equation gives the two loop $\beta$ and $\eta$. Let us reconsider the crucial step of the calculation of $\eta$ (the case of the $\beta$-function is analogous). We have checked that at any finite value of $k$ Eq. (19) yields a wrong coefficient of $\left(\lambda /\left(16 \pi^{2}\right)\right)^{2}$ for $\eta$. This wrong coefficient does not depend on the scale $k$ which means that it stays unchanged in the limit $k \rightarrow 0$. The situation is different if one tries to compute the right hand side of Eq. (19) directly at $k=0$ because of the appearance of some singularities. In fact, in the case considered above, the exponential term $\exp \left(-\frac{p^{2}}{k^{2}}\right)$ in Eq. (19) shows a singularity that corresponds to a $\delta$-function which, in turn, leads to the correct value of $\eta$. Therefore by exchanging the operations of taking the limit $k \rightarrow 0$ and performing the integrals, one gets different results. So either one concludes that the results in Eqs. (21) and (33) are purely accidental or one has to take them as a hint that the flow equation here considered has a peculiar limit $k \rightarrow 0$ which contains some correct features of the exact theory.

In principle one could perform the same analysis by starting with the proper time flow equation regulated by the $m$-dependent smooth cut-off. It is however very problematical to solve the various integrals in this case both in the limit $k \rightarrow 0$ and at finite $k$. It is instead quite simple to check that the $m$-dependent flow equations for the various $n$-point functions become again the exponential flow equations considered above when the limit $m \rightarrow \infty$ is considered and therefore, even in this case, the same results for $\eta$ and $\beta$ are obtained.

Another remark concerns the addition of a mass term in Eqs. (9) and (15). The effect on the exponential flow equations is the appearance of a squared mass term $M^{2}$ in the two-point function in Eq. (13), which can always be 
disentangled from the momentum dependent part of the two-point function due to the exponential form of $D_{i, j}^{y}(p)$. Therefore the flow equations will contain multiplicative factors like $\exp \left(-M^{2} / k^{2}\right)$ and it is not difficult to realize that in this case the limit $k \rightarrow 0$ corresponds to a vanishing $\beta$-function. Clearly this is an indication that in the massive case, when $k$ is approaching zero and is much smaller than the mass, the flow has stopped and the various running quantities have become constant. Then the approach used here yields the perturbative results only if the flow is confined to the critical surface which means that the mass counterterm is fixed in such a way that the theory is massless.

\section{Proper time and exact flow}

In order to have a clearer picture of the differences between the ERG and the PTRG, in this Section we follow [⿴囗十, where a full derivation of the ERG from the fundamental path integral is described, and see to which extent it is possible to reformulate this argument for the PTRG. Let us briefly recall the main points presented in $\mathbb{4}$. In the following the IR regulator is indicated as $H(k ; x, y)$ instead of $R_{k}$ because, as we shall see, they have quite different features. The connected Green function generator $W_{k}$, which depends on the two sources $J(x)$ and $H(k ; x, y)$, is (in the following the integration over the spatial coordinates will be indicated with a center dot)

$$
\exp \left(W_{k}[J, H]\right)=\int \mathcal{D} \phi \exp \left(-S+J \cdot \phi-\frac{1}{2} \phi \cdot H \cdot \phi\right)
$$

and, as usual:

$$
\begin{gathered}
\frac{\delta W_{k}}{\delta J}=\langle\phi\rangle=\varphi \\
\frac{\delta^{2} W_{k}}{\delta J \delta J}=\langle\phi \phi\rangle-\varphi \varphi, \quad \frac{\delta W_{k}}{\delta H}=-\frac{1}{2}\langle\phi \phi\rangle
\end{gathered}
$$

where we have defined

$$
\langle A\rangle=\exp \left(-W_{k}[J, H]\right) \int \mathcal{D} \phi A \exp \left(-S+J \cdot \phi-\frac{1}{2} \phi \cdot H \cdot \phi\right)
$$

The two sources $J(x)$ and $H(k ; x, y)$ (from now on the $x$ and $y$ dependence

of $H$ will not be explicitly displayed in order to have a simpler notation) are independent and we can consider variations of $W_{k}$ with respect to one of them 
while keeping fixed the other. Now we consider the partial Legendre transform of $W_{k}$ with respect to $J$

$$
\widetilde{\Gamma}_{k}[\varphi, H]=-W_{k}[J, H]+J \cdot \varphi
$$

where $\varphi$ has been defined in Eq. (35) and $J$ in the r.h.s. of Eq. (38) is expressed as

$$
J=J[\varphi, H]
$$

which is obtained by inverting Eq. (35).

Then, as usual we can compute some functional derivatives

$$
\begin{gathered}
\frac{\delta \widetilde{\Gamma}_{k}}{\delta \varphi}=-\frac{\delta W_{k}}{\delta J} \cdot \frac{\delta J}{\delta \varphi}+\varphi \cdot \frac{\delta J}{\delta \varphi}+J=J \\
\frac{\delta^{2} \widetilde{\Gamma}_{k}}{\delta \varphi \delta \varphi}=\frac{\delta J}{\delta \varphi}=\left(\frac{\delta^{2} W_{k}}{\delta J \delta J}\right)^{-1}
\end{gathered}
$$

The average effective action $\Gamma_{k}$ is defined as

$$
\Gamma_{k}[\varphi, H]=\widetilde{\Gamma}_{k}[\varphi, H]-\frac{1}{2} \varphi \cdot H \cdot \varphi
$$

and it is easy to check that the source $J$ which is expressed through Eq. (39) is to be considered as a $k$-dependent term, whereas $\varphi$ is an independent variable. Then, the second functional derivative is

$$
\frac{\delta^{2} \Gamma_{k}}{\delta \varphi \delta \varphi}=\frac{\delta^{2} \widetilde{\Gamma}_{k}}{\delta \varphi \delta \varphi}-H
$$

where as stated before the derivation with respect to $\varphi$ is done with $H$ fixed. Recalling that $H$ depends on $k$, it follows:

$$
\begin{aligned}
\partial_{k} \widetilde{\Gamma}_{k} & =-\partial_{k} W_{k}-\frac{\delta W_{k}}{\delta J} \cdot \partial_{k} J+\varphi \cdot \partial_{k} J \\
& =-\partial_{k} W_{k}=\frac{1}{2} \operatorname{Tr}\left(\partial_{k} H \cdot\langle\phi \phi\rangle\right)
\end{aligned}
$$

For the latter equation it must be noticed that the source $J$ which is expressed through Eq. (39) is to be considered as a $k$-dependent term, whereas $\varphi$ is an independent variable. Then for $\Gamma_{k}$ we get:

$$
\partial_{k} \Gamma_{k}=\partial_{k} \widetilde{\Gamma}_{k}-\frac{1}{2} \varphi \cdot \partial_{k} H \cdot \varphi=\frac{1}{2} \operatorname{Tr}\left\{\partial_{k} H \cdot[\langle\phi \phi\rangle-\varphi \varphi]\right\}
$$




$$
=\frac{1}{2} \operatorname{Tr}\left\{\partial_{k} H \cdot\left(\frac{\delta^{2} \Gamma_{k}}{\delta \varphi \delta \varphi}+H\right)^{-1}\right\} .
$$

This is the flow equation for $\Gamma_{k}$. Moreover the specific requirement that $H(k=$ $0)=0$ implies that the functionals $W_{k}$ and $\Gamma_{k}$ become equal to the connected Green function generator and to the effective action respectively, as can be directly checked from their definitions in Eqs. (34), and (42).

Furthermore, if the regulator, evaluated at the UV cut-off $H(k=\Lambda)$ for very large $\Lambda$, is actually divergent, then at that scale the average effective action reduces to the original action

$$
\Gamma_{\Lambda}=S
$$

To prove Eq. (47) let us consider the following shift in the functional integral in Eq. (34)

$$
\phi=\varphi+\phi^{\prime}
$$

where $\varphi$ is a background field which we choose equal to the expectation value of the field defined in Eq. (35)

$$
\begin{gathered}
\exp \left(W_{k}\right)=\int \mathcal{D} \phi^{\prime} \exp \left(-S\left[\phi^{\prime}+\varphi\right]+J \cdot\left(\phi^{\prime}+\varphi\right)-\frac{1}{2}\left(\phi^{\prime}+\varphi\right) \cdot H \cdot\left(\phi^{\prime}+\varphi\right)\right) \\
=\int \mathcal{D} \phi^{\prime} \exp \left(-S\left[\phi^{\prime}+\varphi\right]+J \cdot \phi^{\prime}-\frac{1}{2} \phi^{\prime} \cdot H \cdot \phi^{\prime}\right) \\
\times \exp \left(J \cdot \varphi-\frac{1}{2} \varphi \cdot H \cdot \varphi\right) \exp \left(-\frac{1}{2}\left(\phi^{\prime} \cdot H \cdot \varphi+\varphi \cdot H \cdot \phi^{\prime}\right)\right)
\end{gathered}
$$

By deriving (42) one gets

$$
\frac{\delta \Gamma_{k}}{\delta \varphi}=J-\frac{1}{2} H \cdot \varphi-\frac{1}{2} \varphi \cdot H
$$

and therefore, by inserting (42) and (49) one gets

$$
\exp \left(-\Gamma_{k}\right)=\int \mathcal{D} \phi^{\prime} \exp \left(-S\left[\phi^{\prime}+\varphi\right]+\frac{\delta \Gamma_{k}}{\delta \varphi} \cdot \phi^{\prime}-\frac{1}{2} \phi^{\prime} \cdot H \cdot \phi^{\prime}\right)
$$

If $H$ diverges in the limit $k=\Lambda \rightarrow \infty$, the term $\exp \left(\phi^{\prime} \cdot H \cdot \phi^{\prime} / 2\right)$ behaves like a functional delta $\delta\left[\phi^{\prime}\right]$ in that limit, and therefore Eq. (47) follows from Eq. (50). 
Let us now turn to the proper time flow equation and reconsider again the problem with a different input. As a first remark we note that in Eq. (35) $\varphi$ is defined by a functional derivative of $W_{k}$ with respect to $J$ and keeping $H$ fixed. We keep the same definition of $\varphi$, but then we evaluate $W_{k}$ and $\delta W_{k} / \delta J$ for a particular source $H$, namely

$$
H=\bar{H}(\varphi, k ; x, y)
$$

whose explicit form will be given later ( as before the $x$ and $y$ dependence in $\bar{H}$ will not be displayed). To keep track of this important difference with the previous case we shall use the new notation $\bar{W}, \bar{\Gamma} \widetilde{\bar{\Gamma}}$ (for simplicity the dependence on the scale $k$ is omitted), instead of $W_{k}, \Gamma_{k}$ and $\widetilde{\Gamma}_{k}$. Obviously with this choice Eq. (35) is no longer a simple definition of $\varphi$ and it has become an equation which defines $\varphi$ only implicitly. An explicit expression of $\varphi$ would be obtained only solving the equation. Nevertheless we define $\varphi$ as the solution of this equation, that is $\varphi(x)=\varphi[J, \bar{H}(\varphi, k)]$ and $J$ can be obtained by inverting the latter

$$
J=J[\varphi, \bar{H}]
$$

Moreover, as in the derivation considered before, $J$ depends on $k$ through its dependence on $\bar{H}$ in Eq. (52).

We can go on and define as before

$$
\begin{gathered}
\widetilde{\bar{\Gamma}}[\varphi, \bar{H}]=-\bar{W}[J, \bar{H}]+J \cdot \varphi \\
\bar{\Gamma}=\widetilde{\bar{\Gamma}}-\frac{1}{2} \varphi \cdot \bar{H} \cdot \varphi .
\end{gathered}
$$

Again in these two equations $J$ is to be expressed through Eq. (52).

It is clear that both $\widetilde{\bar{\Gamma}}$ and $\bar{\Gamma}$ due to Eq. (51) depend only on $\varphi$ and $k$. Then it must be remarked that many equations which hold for a simple $\varphi$ independent source $H$, here are no longer valid. For instance if we evaluate $\delta \widetilde{\bar{\Gamma}} / \delta \varphi$ (we intend here the full variation of $\widetilde{\bar{\Gamma}}$ with respect to $\varphi$, including the implicit dependence through $\bar{H}$ ) we get

$$
\frac{\delta \widetilde{\bar{\Gamma}}}{\delta \varphi}=-\frac{\delta \bar{W}}{\delta J} \cdot \frac{\delta J}{\delta \varphi}-\frac{\delta \bar{W}}{\delta \bar{H}} \cdot \frac{\delta \bar{H}}{\delta \varphi}+\frac{\delta J}{\delta \varphi} \cdot \varphi+J=J-\frac{\delta \bar{W}}{\delta \bar{H}} \cdot \frac{\delta \bar{H}}{\delta \varphi}
$$

Therefore it is no longer possible to recover the simple relation in Eq. (41) between $\delta^{2} \widetilde{\bar{\Gamma}} /(\delta \varphi \delta \varphi)$ and $\delta^{2} \bar{W} /(\delta J \delta J)$ (which, as already discussed when we 
introduced $\bar{H}$, is the second functional derivative of $\bar{W}$ with respect to $J$ but keeping $\bar{H}$ fixed).

With this caveat we derive the flow equation

$$
\begin{gathered}
\partial_{k} \widetilde{\bar{\Gamma}}=\frac{1}{2} \operatorname{Tr}\left(\partial_{k} \bar{H} \cdot\langle\phi \phi\rangle\right) \\
\partial_{k} \bar{\Gamma}=\frac{1}{2} \operatorname{Tr}\left[\partial_{k} \bar{H} \cdot\left(\frac{\delta^{2} \bar{W}}{\delta J \delta J}\right)\right] .
\end{gathered}
$$

At this point the proper time flow equation is easily obtained from Eq. (57). In fact now we choose the explicit form of the source $\bar{H}$ to be

$$
\bar{H}(x, y)=2 \int_{0}^{k} \mathrm{~d} q\left\{\left[\bar{W}_{q}^{\prime \prime}\left(x, x^{\prime}\right)\right]^{-1} \cdot \frac{1}{q} \exp \left(-\frac{1}{Z_{q} q^{2}} \bar{\Gamma}_{q}^{\prime \prime}\left(x^{\prime}, y\right)\right)\right\}
$$

where we have used the following notations

$$
\left[\bar{W}_{q}^{\prime \prime}\left(x, x^{\prime}\right)\right]^{-1}=\left[\left(\frac{\delta^{2} \bar{W}}{\delta J(x) \delta J\left(x^{\prime}\right)}\right)_{J=J[\varphi, \bar{H}(\varphi, q)]}\right]^{-1}
$$

and

$$
\bar{\Gamma}_{q}^{\prime \prime}\left(x^{\prime}, y\right)=\left(\frac{\delta^{2} \bar{\Gamma}}{\delta \varphi\left(x^{\prime}\right) \delta \varphi(y)}\right)_{\bar{H}=\bar{H}(\varphi, q)}
$$

and the dot in Eq. (58) indicates integration with respect to $x^{\prime}$. For the sake of clarity the dependence on the scale $q$ is indicated explicitly in Eqs. (59) and (60). Note again that in Eq. (58) $\left[\bar{W}_{q}^{\prime \prime}\left(x, x^{\prime}\right)\right]^{-1}$ is kept explicitly because it cannot be simply replaced with the second derivative of $\bar{\Gamma}$. By replacing Eq. (58) into Eq. (57) one gets

$$
k \partial_{k} \bar{\Gamma}=\operatorname{Tr}\left[\exp \left(-\frac{1}{Z_{k} k^{2}} \bar{\Gamma}_{k}^{\prime \prime}\right)\right]
$$

which is precisely the flow equation in Eq. (6).

Unfortunately in the procedure outlined above the explicit form of $\bar{H}$ is always hidden and, in general, very difficult to determine because it requires one to solve Eq. (58). However, at least for a simple example, it is possible to show how the procedure works. We consider here, as an application, the free theory which can be fully solved. We first remind one that for the free 
theory the exact computation of the path integral leads to the following relation between the classical and the effective action

$$
\Gamma=S+\frac{1}{2} \operatorname{Tr}\left(\log \left(S^{\prime \prime}\right)\right)
$$

where the second derivative of the action is field independent and in the momentum space reads $S^{\prime \prime}=p^{2}+M^{2}$. The divergent term $(1 / 2) \operatorname{Tr}\left(\log S^{\prime \prime}\right)$ is usually cancelled by a normalization factor in the path integral and the conclusion is that $\Gamma=S$. Let us go back to the proper time flow equation which is solved straightforwardly

$$
\Gamma_{k}-S=\operatorname{Tr}\left(\int_{\Lambda}^{k} \frac{\mathrm{d} x}{x} e^{-S^{\prime \prime} / x^{2}}\right)
$$

We have used the boundary condition that at $k=\Lambda$ the running effective action $\Gamma_{\Lambda}$ is equal to $S$ and it is easy to realize that in the right hand side of Eq. (63) the second derivative of the running effective action is $S^{\prime \prime}$ because the flow, due to the boundary condition, modifies $\Gamma_{k}$ only by a field independent term which cannot affect $\Gamma_{k}^{\prime \prime}$ and therefore $\Gamma_{k}^{\prime \prime}=S^{\prime \prime}$. Let us consider the IR limit in Eq. (63) putting $k=0$ and, by identifying the running action at $k=0$ with the effective action $\Gamma$ we get

$$
\Gamma=S-\frac{1}{2} \operatorname{Tr}\left(\int_{1 / \Lambda^{2}}^{\infty} \frac{\mathrm{d} \tau}{\tau} e^{-\tau S^{\prime \prime}}\right)
$$

which is essentially equivalent to Eq. (62) and this time the cancellation of the infinite constant (when the UV cut-off $\Lambda$ is sent to infinity the integral is divergent) in Eq. (64) is obtained by simply modifying the boundary condition of the flow equation with the addition of the proper counterterm to the classical action at the scale $\Lambda$. Once $\Gamma_{k}$ is determined, we follow the same procedure used to determine Eq. (69) and recalling that $\Gamma_{k}^{\prime \prime}=S^{\prime \prime}$ for this particular problem, we get

$$
\left[\bar{W}_{k}^{\prime \prime}\right]^{-1}=S^{\prime \prime}+\bar{H}_{k}
$$

where we have explicitly indicated the scale dependence of $\bar{H}$. Now we have all the ingredients to solve Eq. (58) which is equivalent to the differential equation

$$
k \frac{\partial \bar{H}_{k}}{\partial k}=2\left[\left(S^{\prime \prime}+\bar{H}_{k}\right) \exp \left(-\frac{S^{\prime \prime}}{k^{2}}\right)\right]
$$


together with the condition $\bar{H}_{k=0}=0$. Thus we finally get the solution

$$
\bar{H}_{k}=S^{\prime \prime}\left[\exp \left(\int_{1 / k^{2}}^{\infty} \frac{\mathrm{d} \tau}{\tau} e^{-\tau S^{\prime \prime}}\right)-1\right]=S^{\prime \prime}\left[\exp \left(\Gamma\left(0, \frac{S^{\prime \prime}}{k^{2}}\right)\right)-1\right]
$$

where we have expressed the integral in terms of the incomplete Gamma function $\Gamma(a, b)$. Then, for the non-interacting theory the solution $\bar{H}$ has a simple field independent form which was indicated simply as $H$ at the beginning of this Section. In this case, as discussed before, $\bar{H}$ has exactly the same role of the regulators $R_{k}$ employed for the Exact Renormalization Group flow and therefore the same interpretation of smooth cut-off of the IR modes (clearly even the flow equation is of the same form, as can be easily checked).

Let us come back to the comparison with the ERG. Clearly Eq. (58) is not a simple definition of the regulator, but it is rather an implicit equation for $\bar{H}$ whose resolution is in practice equivalent to solve Eq. (61). This poses a serious limitation on our procedure and in particular on the possibility of showing that $\bar{\Gamma}$ interpolates between the bare action in the UV and the full effective action in the IR. In fact according to the derivation outlined above, $\bar{\Gamma}$ is practically defined through the flow in Eq. (61) and $\bar{H}$ through Eq. (58). The latter can be put in the form of a differential equation for $\bar{H}$ by deriving both sides with respect to the scale $k$. Then we are able to choose the boundary condition (BC) for $\bar{\Gamma}$ in the UV and determine, by means of the flow equation, the form of $\bar{\Gamma}$ in the IR region. In principle it should not be possible to take the $\mathrm{BC}$ in the IR and determine the action in the UV but in practice it is still possible, provided one puts sufficient constraints on the most general form of $\bar{\Gamma}$. Since $\bar{H}$ is related to $\bar{\Gamma}$ through Eq. (54), the BC chosen for $\bar{\Gamma}$ is related to the $\mathrm{BC}$ of the differential equation for $\bar{H}$.

However, here comes the first important difference with respect to the ERG where the explicit form of $H$ is given as an input. Here the explicit form of $\bar{H}$ is given by the solution of Eq. (58), which means that we do not have the freedom to take for instance in the UV region an $\bar{H}$ which diverges to get the condition $\bar{\Gamma}=S$ at the UV scale $\Lambda$, as in the case of the ERG (see Eq. (47)). The only possibility left is to check that if we assume that at the scale $\Lambda$ the relation $\bar{\Gamma}=S$ holds, then Eq. (58) admits a divergent $\bar{H}$ as a solution. In this case we can conclude that the two $\mathrm{BC} \bar{\Gamma}=S$ and infinite $\bar{H}$ in the UV are consistent.

If one is interested in considering the $\mathrm{BC}$ in the IR instead of the UV, the same problem appears. For the ERG the cutoff $H$ vanishes at $k=0$ and this 
condition insures that the running action tends to the effective action when $k \rightarrow 0$. In our case, since the form of $\bar{H}$ is not explicitly available, we can choose the full effective action as $\mathrm{BC}$ for $\bar{\Gamma}$ at $k=0$, insert it into Eq. (58) and check whether this equation consistently admits the condition $\bar{H}=0$ at $k=0$ or not.

Actually we are able to check the consistency of these BC for $\bar{\Gamma}$ and $\bar{H}$ both in the UV and in the IR. Let us first consider the UV case. We note that if $\bar{H}$ diverges for $k=\Lambda$ and $\Lambda \rightarrow \infty$ then, by following the same steps discussed above for $H$, we find that Eq. (47) holds even in this case. From Eq. (47) we can also compute the second derivative of $\bar{W}_{q}$ which, by definition, is given by $\bar{W}_{q}^{\prime \prime}=\delta \varphi / \delta J$. In fact if we use Eq. (47) to replace $\bar{\Gamma}$ in Eq. (54) we get an expression for $\bar{W}_{q}$ which can be derived with respect to the source $J$, keeping $\bar{H}$ fixed. By deriving once and recalling that this derivative must be equal to the field $\varphi$ by definition, one gets

$$
\frac{\delta S}{\delta \varphi}=J-\varphi \cdot \bar{H}
$$

By deriving once again Eq. (68) with respect to $J$ with $\bar{H}$ fixed we recover the desired expression for $\bar{W}_{q}^{\prime \prime}$ in the UV limit:

$$
\left[\bar{W}_{q}^{\prime \prime}\right]^{-1}=S^{\prime \prime}+\bar{H}
$$

So far we have seen that if $\bar{H} \rightarrow \infty$ when $k=\Lambda$ and $\Lambda \rightarrow \infty$ then Eqs. (47) and (69) hold. Now we can check that these relations are consistent with Eq. (58). In the UV limit the upper extremum of the integral in the right hand side of Eq. (58) tends to infinity and we analyze the region of very large values of $q$ where the exponential is substantially close to one and the relevant term in $\left[\bar{W}_{q}^{\prime \prime}\right]^{-1}$ is $\bar{H}$. Then it is easy to realize that Eq. (58) in the limit $k=\Lambda \rightarrow \infty$ is consistent with the divergent solution $\bar{H} \propto \Lambda^{2}$.

Let us consider the IR case. At $k=0$ the range of integration of the variable $q$ in the right hand side of Eq. (58) vanishes but, before concluding that $\bar{H}(\varphi, k=0)=0$ one has to verify that the integrand is not singular at $k=0$. We note that if $\bar{H}(\varphi, k=0)=0$ then, by using the same steps as in the case of the field independent regulator $H$ one gets that $\bar{\Gamma}$ at $k=0$ becomes the effective action and $\bar{W}$ the connected Green function generator. Moreover, at least for a scalar field, the effective action is convex and therefore 
$\bar{\Gamma}^{\prime \prime}$ is non-negative. As a consequence, putting $\bar{H}(\varphi, k=0)=0$ in the right hand side of Eq. (58) means that the exponential has a safe limit when $k \rightarrow 0$ and $\left[\bar{W}_{q=0}^{\prime \prime}\left(x, x^{\prime}\right)\right]^{-1}$ which becomes the inverse propagator is also non-singular and therefore no singularities appear in the integrand at $k=0$. In conclusion $\bar{H}(\varphi, k=0)=0$ is consistent with Eq. (58). Incidentally in the previous Section the limit $k \rightarrow 0$ of the flow equations has been already encountered, at least within the perturbative expansion. There, since the two-point function was evaluated to the lowest order, we had terms like $\exp \left(-p^{2} / k^{2}\right)$ which, in the limit $k \rightarrow 0$ were treated as $\delta$-functions for the momentum $p$. Here in Eq. (58), the same problem can appear for those particular cases in which $\bar{\Gamma}_{q}^{\prime \prime}$ is vanishing. Then again, in the limit $k \rightarrow 0$ (and therefore $q \rightarrow 0$ ) the exponential should be treated as a $\delta$-function. However no singularity affects Eq. (58) because of the other factor in the trace, $\left[\bar{W}_{q}^{\prime \prime}\left(x, x^{\prime}\right)\right]^{-1}$, which, in the limit $k \rightarrow 0(q \rightarrow 0)$ becomes exactly $\bar{\Gamma}_{q=0}^{\prime \prime}$. Therefore the argument of the trace has the form $x \delta(x)$ which is not singular.

So far we have derived the flow in Eq. (61) for $\bar{\Gamma}$ by means of a particular cut-off $\bar{H}$ defined by Eq. (58) and we have checked that we can consistently take as BC of the flow either an infinite $\bar{H}$ with $\bar{\Gamma}=S$ in the UV or $\bar{H}=0$ with $\bar{\Gamma}$ equal to the effective action in the IR. Of course we do not have the freedom to consider both BC in the UV and the IR simultaneously. This clearly indicates the second important difference with the ERG case where there is a suitable cut-off which simultaneously fulfills both conditions, and it represents the main limit of this analysis which, due to the missing link between UV and IR, cannot be used to conclude that the flow in Eq. (61) is exact.

\section{Conclusions}

We conclude with some comments on the results obtained above. We have reformulated and modified the derivation of the ERG flow for the case of the PTRG flow. Since the latter does not belong to the same class of the former, the price we had to pay is that, instead of introducing a previously defined regulator as it happens for $R_{k}$ in the case of the ERG, the regulator $\bar{H}$ comes out as the solution of a very involved equation. We have seen that in the simplest case, which corresponds to the free theory, $\bar{H}$ can be derived explicitly, it is field independent and it is practically equivalent to a regulator 
$R_{k}$, but in the interacting case $\bar{H}$ is field dependent and it is extremely difficult to determine its form. Due to this difficulty, the characteristic properties of $R_{k}$ in the UV and in the IR, which insure the exactness of the ERG, cannot be extended to $\bar{H}$ and the PTRG.

On the other hand we have seen that for the latter flow it is possible to consistently choose for the running action $\bar{\Gamma}$ the $\mathrm{BC} \bar{\Gamma}=S$ in the UV region or, alternatively, take $\bar{\Gamma}$ equal to the effective action in the IR region at $k=0$. Once one of these $\mathrm{BC}$ is chosen one should be able in principle to determine the running action at each value of the scale $k$ by solving the flow equation. This point could be employed, at least in some specific example where some informations on the cut-off $\bar{H}$ can be derived, for instance to study the deviation of the PTRG from the classical action when one chooses the full effective action as an IR BC.

A different kind of indication has been obtained from the perturbative analysis in Section 2. As already noticed, in [19 it is discussed that the diagrammatic structure beyond one loop is lost in the PTRG flow. This means that in a loop expansion, starting at two loop one does not recover the correct weights of the various diagrams. The origin of this problem can be understood if one looks at Eq. (6) and expands the exponential truncating to the linear term in $\Gamma^{\prime \prime} / k^{2}$. Then one can easily recognize that this truncation is equal to the analogous truncation of the expansion of the ERG equation where the cut-off $R_{k}=k^{2}$ is used. So only in the region where $\Gamma^{\prime \prime}<<k^{2}$ the two flow equations have similar features, but since the trace in the right hand side of the equation involves momenta at any scale, there are always regions where the mentioned expansion does not hold and consequently the two flows have different structures. In Section 2 we have checked that the standard perturbative analysis of the flow equation leads to uncorrect results at two loop for the $\beta$-function and the anomalous dimension. It is still possible to extract the two loop results if one considers the limit $k \rightarrow 0$ before performing all integrals on the momenta, which signals the peculiarity of the point $k=0$ for the flow in Eq. (6).

The author acknowledges A. Bonanno and M. Reuter for many helpful discussions and suggestions. 


\section{References}

[1] K.G. Wilson, Phys. Rev. B4, 3174 and 3184, (1971); K.G. Wilson and M.E. Fisher, Phys. Rev. Lett. 28, 240, (1972); K.G. Wilson and J. Kogut, Phys. Rep. 12, 75, (1974).

[2] J. Polchinski, Nucl. Phys. B 231, 269 (1984).

[3] C. Wetterich, Nucl. Phys. B352, 529, (1991); Z. Phys. C57, 451, (1993); C60 461, (1993);

[4] C. Wetterich, Phys Lett. B301, 90, (1993).

[5] M. Bonini, M. D' Attanasio and G. Marchesini, Nucl. Phys. B409, 441, (1993).

[6] U. Ellwanger, Z. Phys. C62, 503, (1994).

[7] T. Morris, Int. J. Mod. Phys. A 9, 2411, (1994); T. Morris, Phys. Lett. B329, 241, (1994).

[8] J. Berges, N. Tetradis and C. Wetterich, Nonperturbative renormalization group flow in quantum field theory and statistical physics, Preprint: MITCTP-2980, HD-THEP-00-26, May 2000 and hep-ph/0005122, submitted to Phys.Rep.

[9] C. Bagnuls and C. Bervillier, Phys. Rept. 348, 91, (2001).

[10] M. Oleszczuk, Z. Phys. C64, 533, (1994).

[11] R. Floreanini and R. Percacci, Phys. Lett. B356, 205, (1995).

[12] S.-B. Liao, Phys. Rev. D53, 2020, (1996); Phys. Rev. D56, 5008, (1997).

[13] B.J. Schaefer and H.J. Pirner, Nucl. Phys. A627, 481, (1997); Nucl. Phys. A660 439, (1999); J. Meyer, G. Papp, H.J. Pirner and T. Kunihiro, Phys. Rev. C61, 035202, (2000); G. Papp, B.J. Schaefer, H.J. Pirner and J. Wambach, Phys. Rev. D61, 096002, (2000); O. Bohr, B.J. Schaefer and J. Wambach, Int. J. Mod. Phys. A16, 3823, (2001); Int. J. Mod. Phys. A16, 2119, (2001); B. J. Schaefer, O. Bohr and J. Wambach, Finite temperature gluon condensate with renormalization group flow equations, Preprint Dec 2001, e-Print Archive: hep-th/0112087; J. Meyer, K. Schwenzer, H.-J. Pirner and A. Deandrea, Phys. Lett. B526, 79, (2002). 
[14] A. Bonanno and D. Zappalà, Phys. Lett. B504, 181, (2001).

[15] M. Mazza and D. Zappalà, Phys. Rev. D 64, 105013, (2001).

[16] D. Zappalà, Phys. Lett. A290, 35, (2001).

[17] D. F. Litim, Phys. Rev. D64, 105007, (2001); JHEP, 0111, 059, (2001);

[18] D. F. Litim and J. M. Pawlowski, Phys. Lett. B516, 197, (2001).

[19] D. F. Litim and J. M. Pawlowski, Perturbation theory and renormalization group equations Preprint: CERN-TH-2001-327, FAU-TP3-01-10, Nov 2001, e-Print Archive: hep-th/0111191.

[20] J. Schwinger, Phys. Rev. 82, 664, (1951).

[21] T. Papenbrock and C. Wetterich, Z. Phys. C65, 519, (1995); T. R. Morris, Nucl. Phys. B458, 477, (1996); M. Bonini, G. Marchesini and M. Simionato, Nucl. Phys. B483, 475, (1997); P. Kopietz, Nucl. Phys. B595, 493, (2001); T. R. Morris and J. F. Tighe, Int. J. Mod. Phys. A16, 2095, (2001).

[22] A. Bonanno and D. Zappalà, Phys. Rev. D57, 7383 (1998); A. Bonanno, V. Branchina, H. Mohrbach and D. Zappalà, Phys. Rev. D60, 065009, (1999). 